This item was submitted to Loughborough's Research Repository by the author.

Items in Figshare are protected by copyright, with all rights reserved, unless otherwise indicated.

\title{
Separatrix crossing in rotation of a body with changing geometry of masses
}

PLEASE CITE THE PUBLISHED VERSION

https://doi.org/10.1137/18M119687X

PUBLISHER

(C) Society for Industrial and Applied Mathematics (SIAM)

VERSION

AM (Accepted Manuscript)

PUBLISHER STATEMENT

This work is made available according to the conditions of the Creative Commons Attribution-NonCommercialNoDerivatives 4.0 International (CC BY-NC-ND 4.0) licence. Full details of this licence are available at: https://creativecommons.org/licenses/by-nc-nd/4.0/

\section{LICENCE}

CC BY-NC-ND 4.0

\section{REPOSITORY RECORD}

Bao, Jinrong, and Anatoly Neishtadt. 2019. "Separatrix Crossing in Rotation of a Body with Changing Geometry of Masses". figshare. https://hdl.handle.net/2134/36274. 


\title{
SEPARATRIX CROSSING IN ROTATION OF A BODY WITH CHANGING GEOMETRY OF MASSES*
}

\author{
JINRONG BAO ${ }^{\dagger}$ AND ANATOLY NEISHTADT ${ }^{\dagger \ddagger}$
}

\begin{abstract}
We consider free rotation of a body whose parts move slowly with respect to each other under the action of internal forces. This problem can be considered as a perturbation of the Euler-Poinsot problem. The dynamics has an approximate conservation law - an adiabatic invariant. This allows to describe the evolution of rotation in the adiabatic approximation. The evolution leads to an overturn in the rotation of the body: the vector of angular velocity crosses the separatrix of the Euler-Poinsot problem. This crossing leads to a quasi-random scattering in body's dynamics. We obtain formulas for probabilities of capture into different domains in the phase space at separatrix crossings. ${ }^{1}$
\end{abstract}

Key words. rigid body dynamics, adiabatic invariant, separatrix crossing

AMS subject classifications. 70E20, 70E55, 70H11, 70K55, 70K65

1 Introduction Rotation of a rigid body around its centre of mass is a classical problem in mechanics. The case when parts of the body slowly move with respect to each other can be considered as a perturbation to the case of a rigid body. This problem serves as a model of rotation of deformable celestial bodies (see, e.g., $[8,13]$ ). We consider a free rotation of a body with a prescribed slow motion of parts of the body with respect to each other. This problem is a perturbation of the Euler-Poinsot problem. We use averaging method and adiabatic approximation to give a description of the evolution of rotational motion of a body. In the process of this evolution the vector of angular velocity of the body crosses the separatrix of the Euler-Poinsot problem. Separatrix crossing in systems with slowly varying parameters lead to probabilistic phenomena (see, e.g., [2], Sect. 6.4.7). We obtain formulas for probabilities of capture into different domains in the phase space at separatrix crossing. The considered problem has an essential difference from other problems, for which probabilities at separatrix crossing were previously calculated. Namely, here the system temporarily passes several times near separatrices through several domains in the phase space prior to being finally captured into one of them. We consider examples of passages through separatrices and compare theoretical results with a numerics.

Results of this paper could be useful in study of dynamics of natural and artificial celestial objects. Rotation of a planet, once perturbed by a fast catastrophic event (volcano eruption, impact), then evolves slowly due to redistribution of mass (see review [12] and references therein). Obtained in our paper formulas could be used to estimate probabilities of different outcomes of such an evolution. As for artificial celestial bodies, often enough they are equipped with different appendages (solar panels, antennas, etc.). Deployments of these appendages lead to change of geometry of masses and evolution of rotation (see., e.g. $[11,18,19])$. Such bodies may also contain internal moving masses (there are many examples in [5]). Formulas in our paper can be used for control of the motion and prediction of results of the evolution of rotation.

\footnotetext{
* Submitted to the editors DATE.

$\dagger$ Loughborough University, Loughborough, LE11 3TU, UK (J.Bao@lboro.ac.uk, A.Neishtadt@1boro.ac.uk).

${ }^{\ddagger}$ Space Research Institute, Moscow, 117997, Russia

${ }^{1}$ Preliminary version of this manuscript is deposited in arXiv (1712.05357[nlin.CD] 2018).
} 


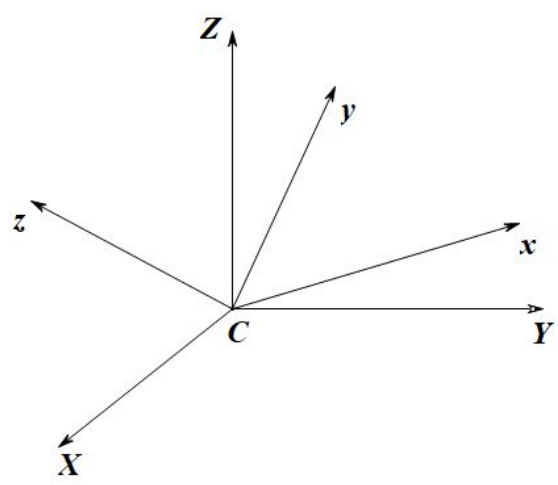

FIG. 1. König's frame and principal axes of inertia frame.

2 Description of the system and equations of motion Consider motion of a system of particles. We assume that there are no external forces acting on these particles. Then the centre of mass $C$ of this system moves with a constant velocity and the angular momentum of this system about $C$ is constant throughout the motion. Consider two frames of reference: a non-rotating frame $C X Y Z$ (König's frame) and the frame $C x y z$ whose axes are principal axes of inertia of the system of particles, Fig. 1. We assume that the motion of the particles with respect to each other is prescribed in advance. An example is the motion of a rigid body and particles which move in a prescribed way with respect to this body. (In this example, as it is traditional in classical mechanics, the rigid body is considered as a system of particles with fixed distances with respect to each other.) One can consider also an object which consists of several rigid bodies moving with respect to each other. Present, following [16], Ch. 1, equations of motion of this system.

The frame $C x y z$ rotates with some angular velocity. Denote $\vec{\omega}$ this angular velocity considered as a vector in the frame $C x y z$. Denote $\vec{G}$ the angular momentum of the moving particles with respect to the point $C ; \vec{G}$ also is considered as a vector in the frame $C x y z$. Let $\hat{I}=\hat{I}(t)$ be the matrix of the inertia tensor of the particles in the axes $C x y z, \vec{g}=\vec{g}(t)$ be the angular momentum about $C$ of the particles in their motion with respect to the frame $C x y z$. Then

$$
\vec{G}=\hat{I} \vec{\omega}+\vec{g}
$$

and

$$
\vec{\omega}=\hat{I}^{-1} \vec{G}-\hat{I}^{-1} \vec{g}
$$

The conservation of the total angular momentum of the system in König's frame implies that

$$
\frac{d \vec{G}}{d t}+\vec{\omega} \times \vec{G}=0
$$

Plugging $\vec{\omega}$ into the equation above, we get the following equation known as Liouville's equation (see, e.g., [13])

$$
\frac{d \vec{G}}{d t}+\hat{I}^{-1}(\vec{G}-\vec{g}(t)) \times \vec{G}=0
$$


In this equation $\hat{I}$ and $\vec{g}$ are prescribed functions of time. We will assume that the relative motion of particles is slow, of order $\varepsilon$, where $\varepsilon>0$ is the small parameter of the problem. Then changes of $\hat{I}$ and $\vec{g}$ with time are slow, of order $\varepsilon$, and $\vec{g}$ is small, of order $\varepsilon$. So, with a slight change of notation $(\hat{I}(t) \rightarrow \hat{I}(\tau), \vec{g}(t) \rightarrow \overrightarrow{\varepsilon g}(\tau))$, and denoting

$$
\vec{f}=\hat{I}^{-1} \vec{g}
$$

we rewrite equation (2.4) as

$$
\frac{d \vec{G}}{d t}+\left(\hat{I}^{-1}(\tau) \vec{G}-\varepsilon \vec{f}(\tau)\right) \times \vec{G}=0, \quad \dot{\tau}=\varepsilon
$$

This system with $f(\tau) \equiv 0$ was studied in [4].

3 Unperturbed system Consider the unperturbed system, i.e. system (2.6) for $\varepsilon=$ $0, \tau=$ const. We get the Euler-Poinsot problem. It can be considered in several representations: on the Poinsot ellipsoid (the ellipsoid of a constant kinetic energy), on the sphere of a constant magnitude of the angular momentum vector, and in the phase cylinder in AndoyerDeprit variables. We will use the last two representations. Denote $A, B$, and $C$ the principal moments of inertia of the body, $A$ corresponds to $C x$ axis, etc. We assume that principal moments of inertia are prescribed functions of the parameter $\tau(A=A(\tau)$, etc.), and their values never coincide. Without loss of generality, assume that $A>B>C$. Vectors $\vec{\omega}=\left(\omega_{1}, \omega_{2}, \omega_{3}\right)$ and $\vec{G}=\left(G_{1}, G_{2}, G_{3}\right)$ are related as $\vec{\omega}=\left(\frac{G_{1}}{A}, \frac{G_{2}}{B}, \frac{G_{3}}{C}\right)$. The sphere $|\vec{G}|=$ const in coordinates $\left(G_{1}, G_{2}, G_{3}\right)$ has the equation

$$
G_{1}^{2}+G_{2}^{2}+G_{3}^{2}=G^{2}
$$

where $G=|\vec{G}|$. Trajectories of the vector $\vec{G}$ on this sphere (see Fig. 2) are isolines of kinetic energy $H_{0}$ of rotation of the body,

$$
H_{0}=\frac{1}{2}\left(\frac{G_{1}^{2}}{A}+\frac{G_{2}^{2}}{B}+\frac{G_{3}^{2}}{C}\right)
$$




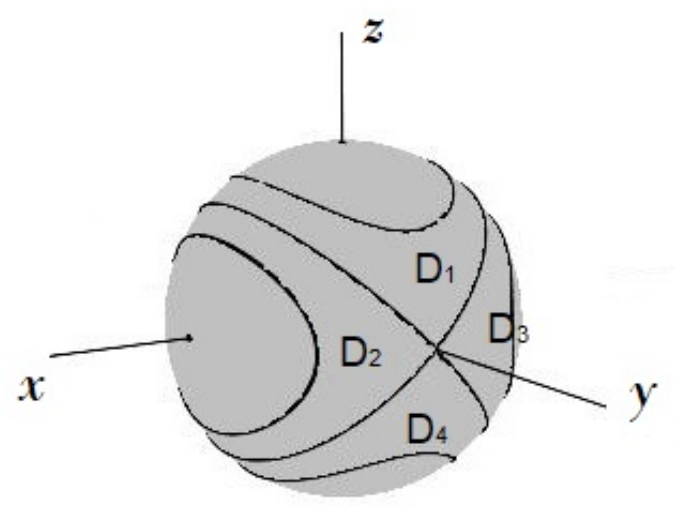

FIG. 2. Rigid body dynamics on the angular momentum sphere.

Let us take direction of the vector $\vec{G}$ as the positive direction of $C Z$ axis (note that $\vec{G}$ is a constant vector in the absolute space). We will use the Andoyer-Deprit variables $L, l$, where $L$ is the projection of $\vec{G}$ onto the axis corresponding to the moment of inertia $C$, and $l$ is the intrinsic rotation angle of the body (Fig. 3).

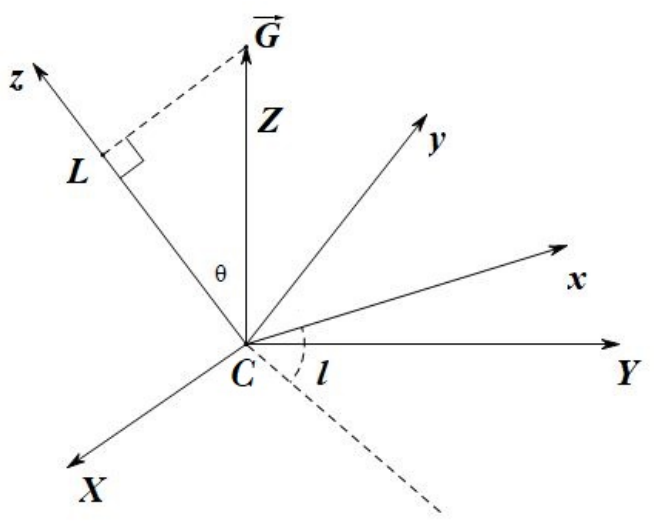

FIG. 3. Andoyer-Deprit variables.

These variables are related to the components of the angular momentum vector as fol- 
lows:

$$
G_{1}=G \sin \theta \sin l, G_{2}=G \sin \theta \cos l, G_{3}=L,
$$

where $\sin \theta=\left(\sqrt{G^{2}-L^{2}}\right) / G$.

Dynamics in the Euler-Poinsot problem in Andoyer-Deprit variables is described by the Hamiltonian system with one degree of freedom, where $l, L$ are conjugate canonical variables [6]. Kinetic energy $H_{0}$ is the Hamiltonian:

$$
H_{0}=\frac{1}{2}\left(\frac{\sin ^{2} l}{A}+\frac{\cos ^{2} l}{B}\right)\left(G^{2}-L^{2}\right)+\frac{L^{2}}{2 C} .
$$

The equations of motion of the system are

$$
\dot{l}=\frac{\partial H_{0}}{\partial L}=\left(\frac{1}{C}-\frac{\sin ^{2} l}{A}-\frac{\cos ^{2} l}{B}\right) L, \quad \dot{L}=-\frac{\partial H_{0}}{\partial l}=\frac{1}{2}\left(\frac{1}{B}-\frac{1}{A}\right)\left(G^{2}-L^{2}\right) \sin 2 l .
$$

The phase portrait of this system should be considered in the cylinder $\{l \bmod 2 \pi, L\}$ (the Deprit cylinder). It is shown in the rectangle $\{0 \leq l \leq 2 \pi,-G \leq L \leq G\}$ in Fig. 4.

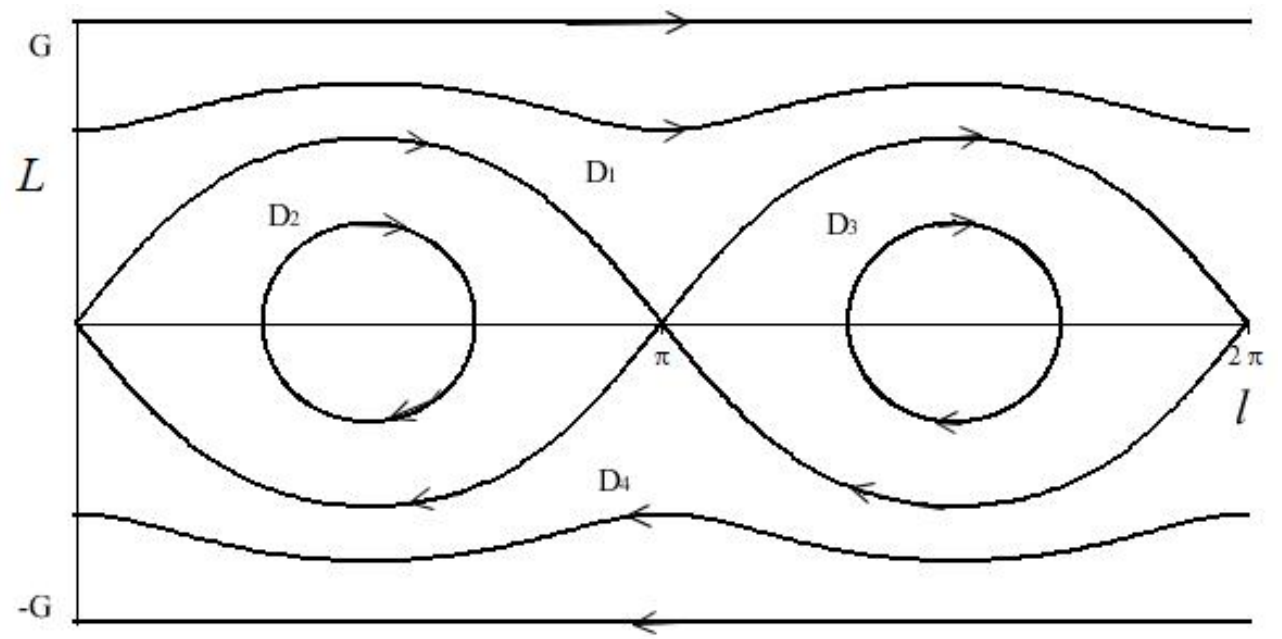

FIG. 4. Phase portrait of the Euler-Poinsot problem.

Stable equilibria in this phase portrait correspond to stationary rotations about $C x$-axis in positive $^{2}$ (for $l=\pi / 2$ ) and negative (for $l=3 \pi / 2$ ) directions. Unstable equilibria correspond to stationary rotations about $C y$-axis in positive (for $l=0$ ) and negative (for $l=\pi$ ) directions. Horizontal lines $L=G$ and $L=-G$ correspond to stationary rotations about $C z$-axis in positive and negative directions, respectively. Values of kinetic energy for stationary rotations about $C x, C y$ and $C z$ axes are $\frac{G^{2}}{2 A}, \frac{G^{2}}{2 B}$ and $\frac{G^{2}}{2 C}$, respectively.

Separatrices divide the phase portrait into domains $D_{1}, D_{2}, D_{3}, D_{4}$ (see Fig. 4). These domains correspond to domains with the same names on the sphere of the constant angular momentum, Fig. 2. Separatrices are determined by the equation

$$
\frac{1}{2}\left(\frac{\sin ^{2} l}{A}+\frac{\cos ^{2} l}{B}\right)\left(G^{2}-L^{2}\right)+\frac{L^{2}}{2 C}=\frac{G^{2}}{2 B}
$$

\footnotetext{
${ }^{2} \mathrm{We}$ consider the anti-clockwise direction as the positive direction of rotation.
} 
which simplifies to

$$
L= \pm G \sin l\left(\frac{\frac{1}{C}-\frac{1}{A}}{\frac{1}{B}-\frac{1}{A}}-\cos ^{2} l\right)^{-1 / 2} .
$$

Action variable $I=I(h, \tau)$ in this phase portrait is defined separately in each domain. In domains $D_{1}$ and $D_{4}$ this is the area between the line $H_{0}=h$ and the line $L=0$ for $0 \leq l \leq 2 \pi$, divided by $2 \pi$. In domains $D_{2}$ and $D_{3}$ this is the area surrounded by the line $H_{0}=h$, divided by $2 \pi$. The formula for $I=I(h, \tau)$ is (see [17], a misprint in this paper is corrected in [9])

$$
I(h, \tau)=\frac{2 G}{\pi \kappa}\left(\frac{1+\kappa^{2}}{\lambda+\kappa^{2}}\right)^{\frac{1}{2}}\left[\left(\lambda+\kappa^{2}\right) \Pi\left(\frac{\pi}{2}, \kappa^{2}, \lambda\right)-\lambda K(\lambda)\right],
$$

where $\kappa$ and $\lambda$ are positive parameters given by

$$
\kappa^{2}=\frac{C(A-B)}{A(B-C)}, \quad \lambda=\frac{(A-B)\left(G^{2}-2 C h\right)}{(B-C)\left(2 A h-G^{2}\right)},
$$

$K$ is the complete elliptic integral of the first kind, $\Pi$ is an elliptic integral of the third kind.

In what follows we need formulas for areas of domains $D_{i}$. They can be obtained as limiting values of $2 \pi I(h, \tau)$ as $h \rightarrow \frac{G^{2}}{2 B}$, or by integrating $L$ given by equation (3.4), or geometrically, from areas on angular momentum sphere. Either way leads to the following results. Area of each of domains $D_{2}, D_{3}$ is

$$
S=4 G \arcsin k, \quad k=\sqrt{\frac{\frac{1}{B}-\frac{1}{A}}{\frac{1}{C}-\frac{1}{A}}} .
$$

Area of each of domains $D_{1}, D_{4}$ is

$$
\tilde{S}=2 \pi G-S=2 G(\pi-2 \arcsin k) .
$$

Areas of the corresponding domains on angular momentum sphere are $G S$ and $G \tilde{S}$.

4 Adiabatic approximation for perturbed system Now consider system (2.6) for $\varepsilon>0, \dot{\tau}=\varepsilon$. We will call $\tau$ "slow time" or just "time" when this does not lead to a mixup. Dynamics of the Andoyer-Deprit variables is described by the Hamiltonian system with the Hamilton's function

$$
H=H(l, L, \tau)=H_{0}(l, L, \tau)+\varepsilon H_{1}(l, L, \tau)
$$

Here $H_{0}$ is the kinetic energy of the body (see (3.3)). The function $H_{1}$ is given by the formula

$$
H_{1}=\vec{f} \cdot \vec{G}=f_{1} \sqrt{G^{2}-L^{2}} \sin l+f_{2} \sqrt{G^{2}-L^{2}} \cos l+f_{3} L
$$

where $f_{i}, i=1,2,3$ are components of the vector $\vec{f}$.

Dynamics of Hamiltonian systems with slowly varying parameters of the form (4.1) can be described in an adiabatic approximation (see, e.g., [2], Sect. 6.4). In particular, the value of the action (3.5) is approximately conserved in the process of motion. The adiabatic approximation in this problem was used in [8] for description of dynamics far from separatrices on the sphere of the angular momentum.

It may happen that in the process of the perturbed motion the phase point in the Deprit cylinder crosses a separatrix of the unperturbed system. Initially, at $t=0$, this phase point is in a domain $D_{i}$, but after some time of order $1 / \varepsilon$. 
Adiabatic approximation can be used up to the separatrix [15]. Thus the moment of separatrix crossing can be approximately found from conservation of the adiabatic invariant as follows. Let $I_{0}$ be initial, at $\tau=0$, value of action. Areas of domains $D_{2,3}$ are functions of $\tau: S(\tau)=4 G \arcsin k(\tau)$. Then the moment $\tau_{*}$ of separatrix crossing is calculated in the adiabatic approximation as the closest to $\tau=0$ root of the equation $S(\tau)=2 \pi I_{0}$. Separatrix crossing leads to an overturn of the body.

\section{Separatrix crossing}

5.1 Separatrix crossing in adiabatic approximation Consider motion of phase points which initially, at $\tau=0$, are in $D_{1}$ at a distance of order 1 from separatrices. We assume that $d S / d \tau>$ const $>0$. Thus areas of domains $D_{2}$ and $D_{3}$ grow, areas of domains $D_{1}$ and $D_{4}$ decay. The phase points approach separatrices at $\tau \approx \tau_{*}$, where $\tau_{*}$ is the moment of separatrix crossing calculated in the adiabatic approximation Sect. 4. We are interested in further motions of these phase points. The averaging method for dynamics with separatrix crossings allows to describe this motion in the adiabatic approximation as follows (see, e.g., [15] and references therein). At the separatrix crossing the phase point can not continue its motion in the domain $D_{1}$ or $D_{4}$ because area of the domain $D_{2} \cup D_{3}$ grows. The phase point continues its motion either in the domain $D_{2}$ or in the domain $D_{3}$. In each case the value of the adiabatic invariant $I$ is equal to $S\left(\tau_{*}\right) /(2 \pi)$. However $S\left(\tau_{*}\right)=2 \pi I_{0}$, where $I_{0}$ is initial, at $\tau=0$, value of action. Thus $I=I_{0}$ in the adiabatic approximation for motions with separatrix crossings.

One can assign certain probabilities to continuations of motion in $D_{2}$ and $D_{3}$. We will calculate these probabilities in Sect. 5.3.

5.2 Change of energy near separatrices In order to study separatrix crossing we need to know asymptotic formulas for change of energy $H_{0}$ in the perturbed system when the phase point moves near unperturbed separatrices (cf. [15]). The energy along the separatrices is $H_{B}=\frac{G^{2}}{2 B}$. Introduce the new function $E=H-H_{B}$. In the perturbed system (2.6) we have

$$
\frac{d E}{d t}=\varepsilon\left(\frac{\partial E}{\partial \tau}+\nabla H \cdot(\vec{f} \times \vec{G})\right) .
$$

Here $\nabla H$ is the gradient of $H$ considered as the function of $G_{1}, G_{2}, G_{3}: \nabla H=\left(G_{1} / A, G_{2} / B, G_{3} / C\right)$.

There are four separatrices, see Fig. 5. Denote the separatrix between $D_{1}$ and $D_{2}$ as $\Gamma_{1,2}$. Similarly we have $\Gamma_{1,3}, \Gamma_{4,2}$ and $\Gamma_{4,3}$.

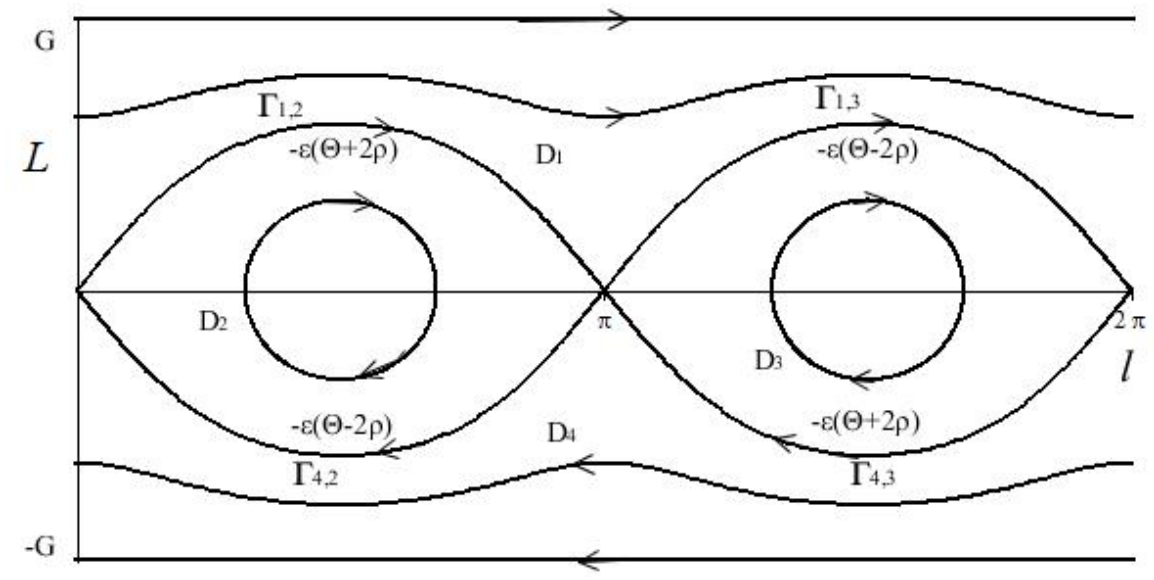

FIG. 5. Separatrices. 
Change $\Delta E$ of value of the function $E$ along a fragment of perturbed trajectory close to a separatrix $\Gamma$ is approximately equal to the integral of $d E / d t$ over $\Gamma$ :

$$
\Delta E \approx \varepsilon \int_{\Gamma}\left(\frac{\partial E}{\partial \tau}\right) d t+\varepsilon \int_{\Gamma} \nabla H \cdot(\vec{f} \times \vec{G}) d t
$$

Estimates of accuracy of such an approximation are contained in [15]. In particular, for motion with $E \sim \varepsilon$ the accuracy is $O\left(\varepsilon^{3 / 2}\right)$. The integrals here are improper ones, as the motion along a separatrix takes infinite time, but they converge.

For calculation of the first integral in (5.1) we use the formula that the $\tau$-derivative of the area, surrounded by separatrices, is equal to the integral of $-(\partial E / \partial \tau)$ over these separatrices (cf. [14]). Denote

$$
\Theta=\Theta(\tau)=\frac{1}{2} \frac{\partial S}{\partial \tau},
$$

where $S$ is the area of each of domains $D_{2,3}$. Thus the first integral in (5.1) is

$$
\int_{\Gamma}\left(\frac{\partial E}{\partial \tau}\right) d t=-\Theta=-\frac{1}{2} \frac{\partial S}{\partial \tau}
$$

for each of separatrices. From Sect. 2 we know that $S=4 G \arcsin k$ with $k^{2}=\frac{\frac{1}{A}-\frac{1}{B}}{\frac{1}{A}-\frac{1}{C}}$. Then

$$
\begin{aligned}
\frac{\partial S}{\partial \tau} & =4 \frac{\partial G \arcsin k}{\partial \tau}=\frac{4 G}{\sqrt{1-k^{2}}} \frac{d k}{d \tau}=\frac{4 G}{\sqrt{1-k^{2}}} \frac{d}{d \tau} \sqrt{\frac{\frac{1}{A}-\frac{1}{B}}{\frac{1}{A}-\frac{1}{C}}} \\
& =2 G \sqrt{\frac{\frac{1}{A}-\frac{1}{C}}{\frac{1}{B}-\frac{1}{C}}} \sqrt{\frac{\frac{1}{A}-\frac{1}{C}}{\frac{1}{A}-\frac{1}{B}}} \cdot \frac{\left(\frac{A^{\prime}(\tau)}{A^{2}}-\frac{B^{\prime}(\tau)}{B^{2}}\right)\left(\frac{1}{A}-\frac{1}{C}\right)-\left(\frac{1}{A}-\frac{1}{B}\right)\left(\frac{A^{\prime}(\tau)}{A^{2}}-\frac{C^{\prime}(\tau)}{C^{2}}\right)}{\left(\frac{1}{A}-\frac{1}{C}\right)^{2}} \\
& =2 G \frac{\left(\frac{A^{\prime}(\tau)}{A^{2}}-\frac{B^{\prime}(\tau)}{B^{2}}\right)\left(\frac{1}{A}-\frac{1}{C}\right)-\left(\frac{1}{A}-\frac{1}{B}\right)\left(\frac{A^{\prime}(\tau)}{A^{2}}-\frac{C^{\prime}(\tau)}{C^{2}}\right)}{\left(\frac{1}{A}-\frac{1}{C}\right) \sqrt{\left(\frac{1}{B}-\frac{1}{C}\right)\left(\frac{1}{A}-\frac{1}{B}\right)}} .
\end{aligned}
$$

Therefore,

$$
\int_{\Gamma} \frac{\partial E}{\partial \tau} d t=-\Theta=-\frac{1}{2} \frac{\partial S}{\partial \tau}=-G \frac{\left(\frac{A^{\prime}(\tau)}{A^{2}}-\frac{B^{\prime}(\tau)}{B^{2}}\right)\left(\frac{1}{A}-\frac{1}{C}\right)-\left(\frac{1}{A}-\frac{1}{B}\right)\left(\frac{A^{\prime}(\tau)}{A^{2}}-\frac{C^{\prime}(\tau)}{C^{2}}\right)}{\left(\frac{1}{A}-\frac{1}{C}\right) \sqrt{\left(\frac{1}{B}-\frac{1}{C}\right)\left(\frac{1}{A}-\frac{1}{B}\right)}} .
$$

Here "prime" denotes the derivative with respect to $\tau$.

Now we compute the second integral in equation (5.1). We have

$$
\nabla H \cdot(\vec{f} \times \vec{G})=\frac{\partial H}{\partial \vec{G}} \cdot(\vec{f} \times \vec{G})=\left(\vec{G} \times \frac{\partial H}{\partial \vec{G}}\right) \cdot \vec{f}=\left(\vec{G} \times \hat{I}^{-1} \vec{G}\right) \cdot \vec{f}=\frac{d \vec{G}}{d t} \cdot \vec{f},
$$

where $\frac{d \vec{G}}{d t}$ is calculated in the unperturbed system.

We know that the point $l=0, L=0$ in the phase portrait Fig. 5 corresponds to a rotation around the axis of the moment of inertia $B$ in the positive direction $\left(G_{2}=G\right)$, while the point $l=\pi, L=0$ corresponds to a rotation around this axis in the negative direction $\left(G_{2}=-G\right)$. Therefore,

$$
\int_{\Gamma_{1,2}} \nabla H \cdot(\vec{f} \times \vec{G}) d t=\int_{\Gamma_{1,2}} \frac{\overrightarrow{d G}}{d t} \cdot \vec{f} d t=\vec{f} \cdot \int_{0}^{\pi} \overrightarrow{d G}=-2 f_{2} G
$$


where $f_{2}$ is the second component of $\vec{f}$. Integral over $\Gamma_{4,3}$ has the same value $-2 f_{2} G$, integrals over $\Gamma_{1,3}$ and $\Gamma_{4,2}$ have value $2 f_{2} G$.

Denote

$$
\rho=\rho(\tau)=f_{2} G .
$$

Then from formula (5.1) and previous calculations we have the following expressions for change $\Delta_{i, j} E$ of value of the function $E$ along a fragment of perturbed trajectory close to a separatrix $\Gamma_{i, j}$ valid in the principal approximation:

$$
\begin{array}{ll}
\Delta_{1,2} E=\varepsilon(-\Theta-2 \rho), & \Delta_{1,3} E=\varepsilon(-\Theta+2 \rho), \\
\Delta_{4,2} E=\varepsilon(-\Theta+2 \rho), & \Delta_{4,3} E=\varepsilon(-\Theta-2 \rho) .
\end{array}
$$

These values of change of the function $E$ for motion near the separatrices are indicated in Fig. 5.

Denote $\Delta_{1}$ and $\Delta_{4}$ changes of $E$ along fragments of perturbed trajectory $O(\varepsilon)$-close to $\Gamma_{1}=\Gamma_{1,2} \cup \Gamma_{1,3}$ and to $\Gamma_{4}=\Gamma_{4,3} \cup \Gamma_{4,2}$, respectively. We have the following expression valid in the principal approximation:

$$
\Delta_{1}=\Delta_{4}=-2 \varepsilon \Theta .
$$

In what follows we use these approximate formulas as exact formulas for change of energy, thus neglecting high order corrections.

5.3 Probabilities of capture into different domains We consider phase points that start their motion at $\tau=0$ in $D_{1}$. These phase points can be captured into $D_{2}$ or $D_{3}$ after separatrix crossing. Initial conditions for capture into different domains are mixed in the phase space. Small, $\sim \varepsilon$, change of initial conditions can change the destination of a phase point after separatrix crossings. Destinations depend also on $\varepsilon$. For a fixed initial condition and $\varepsilon \rightarrow 0$ two destinations replace each in an oscillatory manner. Thus the question about this destination does not have a deterministic answer in the limit as $\varepsilon \rightarrow 0$. It is reasonable to consider capture into a given domain as a random event and calculate the probability of this event. Such an approach was introduced in [10] for scattering of charged particles at separatrix crossing. This approach was rediscovered by many authors. In particular, it was used in [7] for planetary rotations to determine the probability of capture of Mercury in its current resonant regime. A rigorous approach to the definition of the notion of probability in the considered class of problems was suggested in [1]. It is based on the comparison of measures of initial data for captures into different domains. Below we use this approach.

Consider a point $M$ in the domain $D_{1}$ at a distance of order 1 from separatrices. Denote $I_{M}$ the value of action at $M$ at $\tau=0$. Let $\tau_{*}$ be the moment of separatrix crossing in adiabatic approximation for motion with the initial condition at $M, S\left(\tau_{*}\right)=2 \pi I_{M}$. We will consider motion in the time interval $0 \leq \tau \leq K$, where $K=$ const $>\tau_{*}$. Thus, this interval includes the moment of separatrix crossing (in the adiabatic approximation) for the motion with the initial condition at $M$.

Denote $U^{\delta}$ the disc of radius $\delta$ with the centre at $M$. We assume that $\delta$ is small enough, and thus $U^{\delta}$ is in $D_{1}$ at $\tau=0$. Moreover, the moment of separatrix crossing (in the adiabatic approximation) for all motions with initial conditions in $U^{\delta}$ is less than $K$. The set $U^{\delta}$ can be represented as a union of three sets, $U^{\delta}=U_{1}^{\delta, \varepsilon} \cup U_{2}^{\delta, \varepsilon} \cup v_{\varepsilon}$ as follows. The set $U_{i}^{\delta, \varepsilon}, i=1,2$, contains initial conditions of motions for which change of the action $I$ on the time interval $0 \leq \tau \leq K$ tends to 0 as $\varepsilon \rightarrow 0$ (i.e $\sup _{0 \leq t \leq K / \varepsilon}|I(t)-I(0)| \rightarrow 0$ as $\varepsilon \rightarrow 0$ ) and the phase point is in the domain $D_{i}$ at $\tau=K$. It follows from results of [15] that meas $v_{\varepsilon} \rightarrow 0$ as $\varepsilon \rightarrow 0$. I.e. the 
adiabatic approximation works for the majority of initial conditions. Following [1] we call

$$
\operatorname{Pr}_{1, i}(M)=\lim _{\delta \rightarrow 0} \lim _{\varepsilon \rightarrow 0} \frac{\operatorname{meas} U_{i}^{\delta, \varepsilon}}{\text { meas } U^{\delta}}
$$

the probability ${ }^{3}$ of capture of $M$ into domain $D_{i}, i=2,3$. This probability is a function of $\tau_{*}$, which we denote $P_{1, i}\left(\tau_{*}\right), \operatorname{Pr}_{1, i}(M)=P_{1, i}\left(\tau_{*}\right)$ [15]. The phase portrait of the unperturbed system Fig. 5 is different from that in [15]. Nevertheless it is possible to use an approach in [15] to formulate a procedure for calculation of $P_{1, i}\left(\tau_{*}\right)$ as follows.

Phase points from $U^{\delta}$ make many rounds repeatedly crossing the line $l=\pi$ in $D_{1}$ and gradually approaching separatrices. At each such round near the separatrices the value of $E$ decays by about $2 \varepsilon \Theta$. So, at the last arrival to the line $l=\pi$ in $D_{1}$ we have $0<E<2 \varepsilon \Theta$ (in the principal approximation). Phase points from $U^{\delta}$ finish this last round almost simultaneously at $\tau \approx \tau_{*}$. Consider motion of a phase point that starts at $\tau=\tau_{*}$ from the line $l=\pi$ with $E=E_{*}$, where $0<E_{*}<2 \varepsilon \Theta_{*}, \Theta_{*}=\Theta\left(\tau_{*}\right)$. This phase point will make several rounds near separatrices. The change of energy for motion near separatrices will be calculated in the principal approximation by formulas (5.5) with $\Theta=\Theta_{*}, \rho=\rho_{*}$. This construction determines parts of the interval $0<E_{*}<2 \varepsilon \Theta_{*}$ corresponding to captures into $D_{2}$ and $D_{3}$. The ratio to $2 \varepsilon \Theta_{*}$ of length of the part, corresponding to capture into domain $D_{i}$, is equal to the probability $P_{1, i}\left(\tau_{*}\right)$.

Calculate probabilities using such an approach. We will assume that $\rho_{*}>0$. The situation with $\rho_{*}<0$ is reduced to that with $\rho_{*}>0$ by exchange of numbers of domains $D_{2}$ and $D_{3}$.

We will consider two cases.

Case I: $\Theta_{*}>2 \rho_{*}$. A phase point starts at $\tau=\tau_{*}$ from the line $l=\pi$ with $E=E_{*}$, $0<E_{*}<2 \varepsilon \Theta_{*}$. It passes near the separatrix $\Gamma_{1,3}$ during some time interval $\tau_{*} \leq \tau \leq \tau_{1}$ first, and arrives either to the line $L=0$ near $l=0$ in $D_{3}$ or to the line $l=0$ in $D_{1}$. At the end of this pass the value of $E$ is $E_{1}=E_{*}-\varepsilon\left(\Theta_{*}-2 \rho_{*}\right)$. Two sub-cases are possible.

a) If $E_{1}<0$, then at $\tau=\tau_{1}$ the phase point is in $D_{3}$. In further motion it will make rounds near $\Gamma_{4,3} \cup \Gamma_{1,3}$, returning to the line $L=0$ near $l=0$ in $D_{3}$ after each round. The value of $E$ decays by $2 \varepsilon \Theta_{*}$ at each such round. This is a capture into $D_{3}$.

b) If $E_{1}>0$, then at $\tau=\tau_{1}$ the phase point is in $D_{1}$. Then it passes near the separatrix $\Gamma_{1,2}$ during some time interval $\tau_{1} \leq \tau \leq \tau_{2}$, and arrives to the line $L=0$ near $l=\pi$ in $D_{2}$ with $E=E_{2}=E_{1}-\varepsilon\left(\Theta_{*}+2 \rho_{*}\right)=E_{*}-2 \varepsilon \Theta_{*}<0$. In further motion it will make rounds near $\Gamma_{4,2} \cup \Gamma_{1,2}$ returning to $L=0$ near $l=\pi$ in $D_{2}$ after each round. The value of $E$ decays by $2 \varepsilon \Theta_{*}$ at each such round. This is a capture into $D_{2}$.

Thus, if $\Theta_{*}>2 \rho_{*}$, then the probabilities are

$$
P_{1,2}=\frac{\Theta_{*}+2 \rho_{*}}{2 \Theta_{*}}, \quad P_{1,3}=\frac{\Theta_{*}-2 \rho_{*}}{2 \Theta_{*}} .
$$

Case II: $\Theta_{*}<2 \rho_{*}$. Let $k$ be the natural number such that $(2 k-1) \Theta_{*}<2 \rho_{*}<(2 k+1) \Theta_{*}$. A phase point starts at $\tau=\tau_{*}$ from the line $l=\pi$ with $E=E_{*}, 0<E_{*}<2 \varepsilon \Theta_{*}$. During a time interval $\tau_{*} \leq \tau \leq \tau_{1}$, it goes along a path which is close to sequence of segments $\Gamma_{1,3} \cup \Gamma_{1,2}$ and $\Gamma_{4,2} \cup \Gamma_{4,3}$. The path contains $k$ such segments. At $\tau=\tau_{1}$ the phase point is at the line $L=0$ near $l=\pi$ in $D_{2}$ or in $D_{3}$ with $E=E_{1}=E_{*}-2 k \varepsilon \Theta_{*}$. It is in $D_{2}$ if $k$ is odd, and in $D_{3}$, if $k$ is even. Consider, for definiteness, the case when $k$ is odd. The phase point passes near the separatrix $\Gamma_{4,2}$ during some time interval $\tau_{1} \leq \tau \leq \tau_{2}$. At the end of this pass the value of $E$ is $E_{2}=E_{1}-\varepsilon\left(\Theta_{*}-2 \rho_{*}\right)=E_{*}-(2 k+1) \varepsilon \Theta_{*}+2 \varepsilon \rho_{*}$. Two sub-cases are possible.

\footnotetext{
${ }^{3}$ This is the probability density for capture into $D_{i}$.
} 
a) If $E_{2}<0$, then at $\tau=\tau_{2}$ the phase point is in $D_{3}$. In further motion it will make rounds near $\Gamma_{1,2} \cup \Gamma_{4,2}$, returning to $L=0$ near $l=0$ in $D_{2}$ after each round. The value of $E$ decays by $2 \varepsilon \Theta_{*}$ at each such round. This is a capture into $D_{2}$.

b) If $E_{2}>0$, then at $\tau=\tau_{2}$ the phase point is in $D_{4}$. Then it passes near the separatrix $\Gamma_{4,3}$ during some time interval $\tau_{2} \leq \tau \leq \tau_{3}$, and arrives to the line $L=0$ near $l=\pi$ in $D_{3}$ with $E=E_{3}=E_{2}-\varepsilon\left(\Theta_{*}+2 \rho_{*}\right)=E_{*}-(2 k+2) \varepsilon \Theta_{*}<0$. Then it passes near the separatrix $\Gamma_{1,3}$ during some time interval $\tau_{3} \leq \tau \leq \tau_{4}$. At the end of this path $E=E_{4}=E_{3}-\varepsilon\left(\Theta_{*}-2 \rho_{*}\right)=$ $E_{*}-(2 k+3) \varepsilon \Theta_{*}+2 \varepsilon \rho_{*}<2 \varepsilon \Theta_{*}-(2 k+3) \varepsilon \Theta_{*}+2 \varepsilon \rho_{*}=-(2 k+1) \varepsilon \Theta_{*}+2 \varepsilon \rho_{*}<0$.Thus at $\tau=\tau_{4}$ the phase point is at the line $L=0$ near $l=0$ in $D_{3}$. In further motion it will make rounds near $\Gamma_{4,3} \cup \Gamma_{1,3}$ returning to $L=0$ near $l=0$ in $D_{3}$ after each round. The value of $E$ decays by $2 \varepsilon \Theta_{*}$ at each such round. This is a capture into $D_{3}$.

Thus if $(2 k-1) \Theta_{*}<2 \rho_{*}<(2 k+1) \Theta_{*}$ with an odd $k$, then the probabilities are

$$
P_{1,2}=\frac{(2 k+1) \Theta_{*}-2 \rho_{*}}{2 \Theta_{*}}, \quad P_{1,3}=\frac{-(2 k-1) \Theta_{*}+2 \rho_{*}}{2 \Theta_{*}} .
$$

Similar reasoning shows that if $(2 k-1) \Theta_{*}<2 \rho_{*}<(2 k+1) \Theta_{*}$ with an even $k$, then the probabilities are

$$
P_{1,2}=\frac{-(2 k-1) \Theta_{*}+2 \rho_{*}}{2 \Theta_{*}}, \quad P_{1,3}=\frac{(2 k+1) \Theta_{*}-2 \rho_{*}}{2 \Theta_{*}} .
$$

Probabilities for initial conditions from $D_{4}$ can be obtained from the previous formulas by replacement of the index 1 with the index 4 and exchange of indexes 2 and 3 .

6 Examples In this Section we consider two examples of calculation of probabilities and compare theoretical and numerical results. In these examples we consider a system which consists of a rigid body and one or two particles which move with respect to this body. We assume that the principal central moments of inertia of the body are different from each other. The body frame $O^{\prime} \xi \eta \zeta$ is the frame of principal central axes of inertia of the body; $O^{\prime}$ is the centre of mass of the body, the $\xi, \eta$ and $\zeta$-axes correspond, respectively, to the maximal, intermediate and minimal moments of inertia of the body.

6.1 Case of equal probabilities Consider a system which consists of a rigid body and a particle which moves along the axis of the intermediate principal central moment of inertia of the body (axis $O^{\prime} \eta$ ). Then the centre of mass of the system $O$ moves along this axis, and this axis is also the axis of a principal central moment of inertia of the system. We assume that this axis corresponds to the intermediate moment of inertia of the system (this is correct if the mass of the moving particle is not too big). The system frame $O x y z$ is a translation of the body frame $O^{\prime} \xi \eta \zeta$ in the direction of $\eta$-axis. Intermediate moment of inertia $B$ of the system does not change. Maximal and minimal moments of inertia $A$ and $C$ of the system change according to the following formulas.

Let $m$ be mass of the moving particle and $\eta=\eta(\tau)$ be $\eta$-coordinate of this particle in the body frame, $\eta_{0}=\eta(0)$. Let $M$ be the total mass of the system. Then (see Appendix A)

$$
A(\tau)=A(0)+\frac{m(M-m)}{M}\left(\eta^{2}-\eta_{0}^{2}\right), C(\tau)=C(0)+\frac{m(M-m)}{M}\left(\eta^{2}-\eta_{0}^{2}\right)
$$

The velocity vector of the moving particle with respect to the frame of principal central axes of inertia of the system is parallel to the position vector of this particle. Therefore, $\vec{g}=0$, and thus $\vec{f}=\hat{I}^{-1} \vec{g}=0$ and $\rho=f_{2} G=0$. According to (5.7), for particles leaving domain $D_{1}$ we 
have

$$
P_{1,2}=\frac{\Theta_{*}}{2 \Theta_{*}}=\frac{1}{2}, \quad P_{1,3}=\frac{\Theta_{*}}{2 \Theta_{*}}=\frac{1}{2} .
$$

For a numerical illustration of dynamics in the considered case we take $\eta=\eta(\tau)=\eta_{0}+\varepsilon t$, $A(0)=10.0, B(0)=8.0, C(0)=6.0, m=0.1, M=10.0, \eta_{0}=1.0$, and $\varepsilon=0.003$. The initial values of $G_{1}, G_{2}, G_{3}$ are 10.0, 9.0, 8.0, respectively. Fig. 6 and Fig. 7 show the graphs of moments of inertia $A$ and $C$ vs $\tau$. (Note that $B$ is a constant in the considered example.)

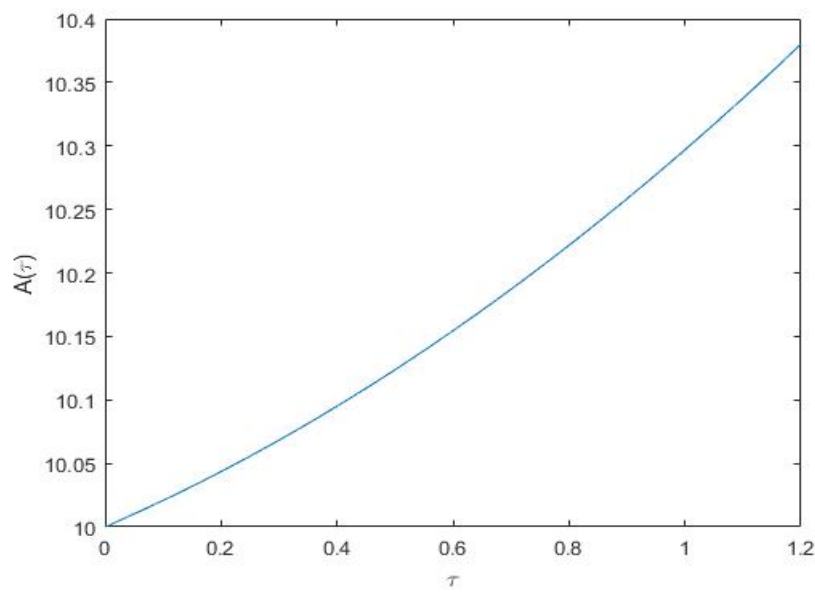

FIG. 6. Graph of the maximal moment of inertia A vs $\tau$.

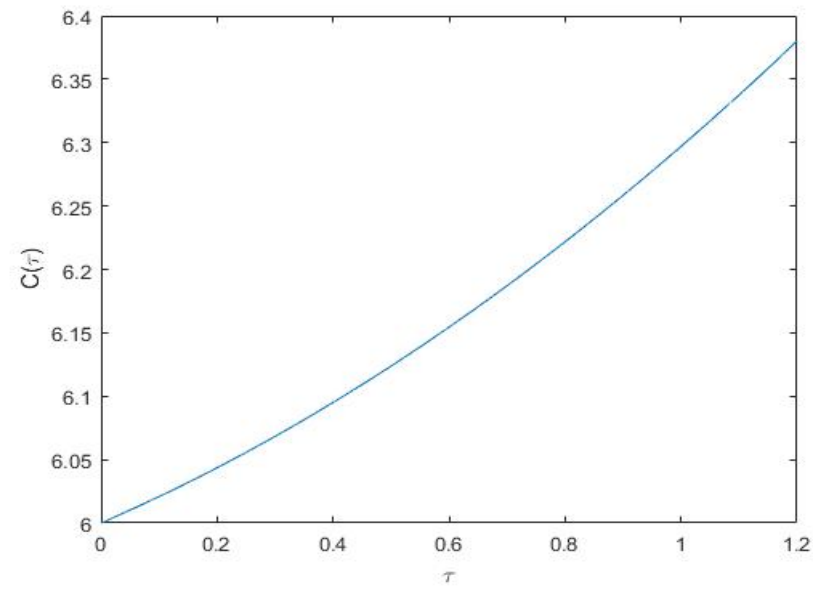

FIG. 7. Graph of the minimal moment of inertia $C$ vs $\tau$.

Fig. 8 and Fig. 9 show changes of $G_{1}$ and $G_{3}$ in dependence on the slow time $\tau$. Recall that $G_{1}=G \sin \theta \sin l$ and $G_{3}=L$. These graphs demonstrate that a) the motion starts in domain $D_{1}$ and b) when $\tau$ is about 0.52 , the phase point crosses the separatrix and stays 
in domain $D_{2}$ after this. Indeed, for the left part of these graphs $G_{1}$ takes both positive and negative values, and $G_{3}$ takes positive values. This corresponds to motion in $D_{1}$. For the right part of these graphs $G_{1}$ takes positive values, and $G_{3}$ takes both positive and negative values. This corresponds to motion in $D_{2}$.

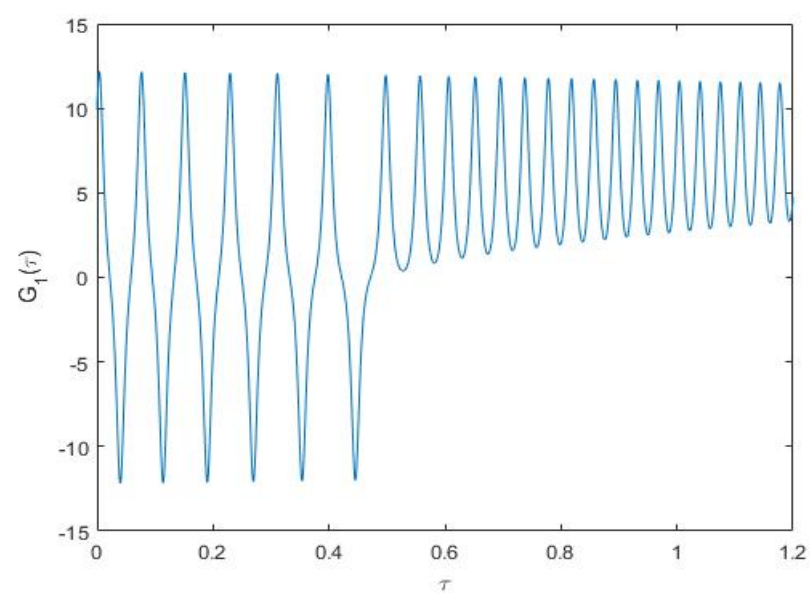

FIG. 8. Graph of $G_{1}$ vs $\tau$.

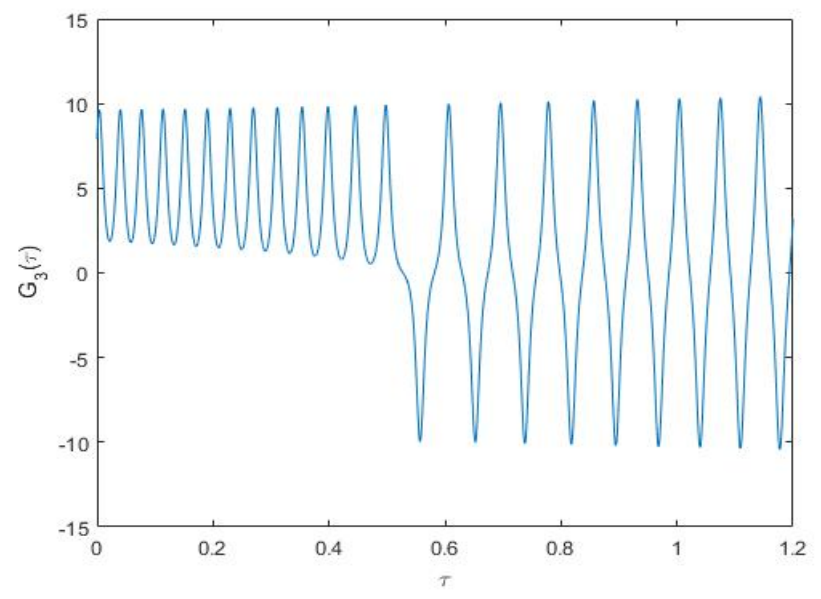

FIG. 9. Graph of $G_{3}$ vs $\tau$.

Dependence of energy $H_{0}$ on slow time $\tau$ is shown in Fig. 10 (blue curve). The horizontal red straight line corresponds to the value of energy on the separatrices $H_{B}=G^{2} /(2 B)=$ const. We see that the energy decays. It is equal to the energy along the separatrices when $\tau$ is around 0.52 , where the crossing happens. 


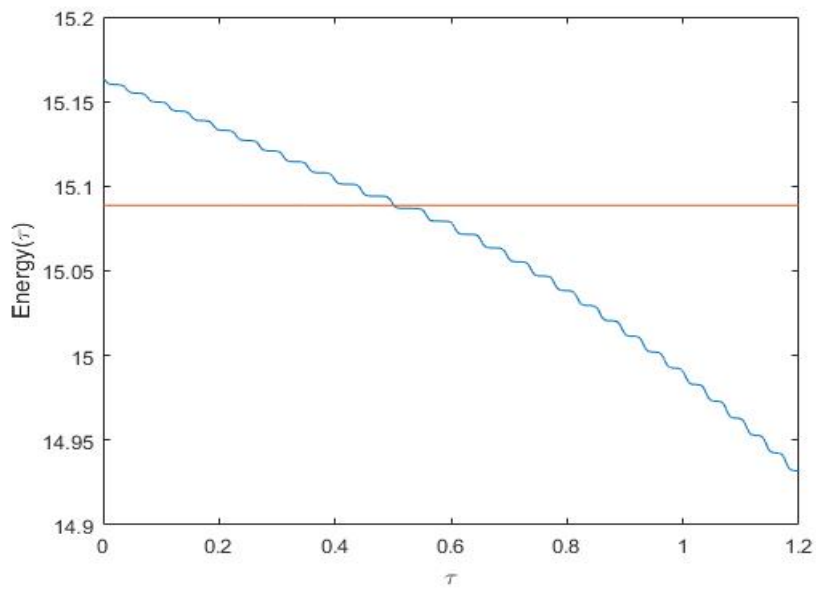

FIG. 10. Graph of energy $H_{0} v s \tau$.

Behaviour of action $I$ in slow time is shown in Fig. 11. We see that the action performs only small oscillations about a constant value. Bigger oscillations of action at $\tau$ about 0.52 correspond to the separatrix crossing.

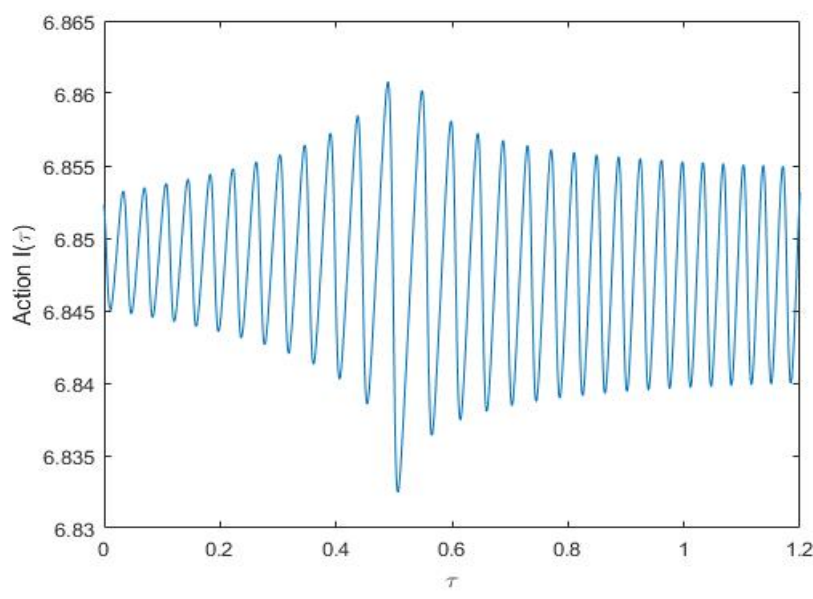

FIG. 11. Graph of action I vs $\tau$.

Fig. 12 shows the graph of area of the domains $D_{2}$ (or $D_{3}$ ) vs $\tau$ (blue curve) and the initial value of action $I$ multiplied by $2 \pi$ (brown horizontal straight line). The blue and brown lines intersect at $\tau=0.5189$. This is the moment of separatrix crossing calculated in adiabatic approximation. This value is in a good agreement with moment of separatrix crossing seen in the previous pictures. 


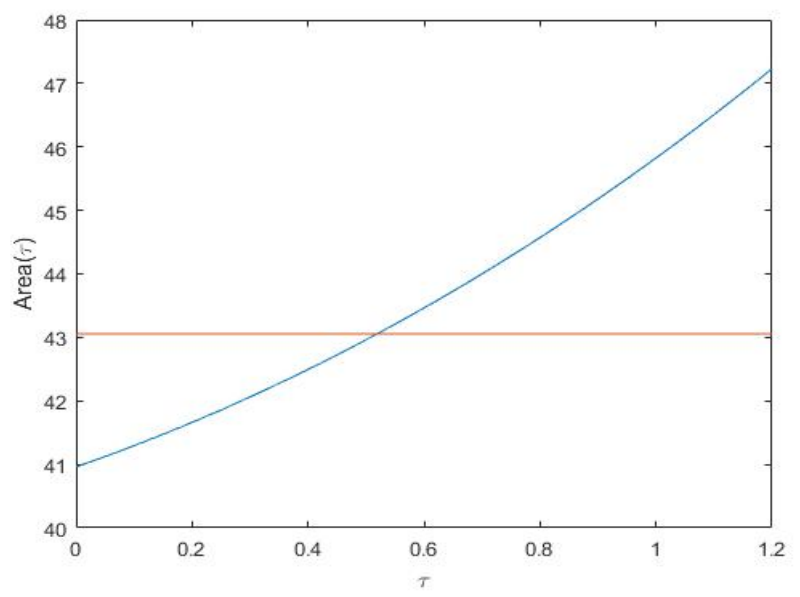

Fig. 12. Graph of area $v s \tau$.

To check the formula for probability we fix $G_{2}=9$ and consider 2000 initial conditions uniformly distributed in the square $9.9 \leq G_{1} \leq 10.1,7.9 \leq G_{3} \leq 8.1$. Trajectories with these initial conditions were captured into $D_{3}$ in 1010 cases and into $D_{2}$ in 990 cases. Thus, numerically calculated probability 0.495 of capture into $D_{2}$ is close to its theoretical value 0.5 .

6.2 Case of non-equal probabilities Consider a system which consists of a rigid body and two particles of equal masses which move in the plane of the maximal and the minimal central moments of inertia of the body. The particles move in a circle with the centre at the centre of mass of the body, and their positions are symmetric with respect to the centre of this circle. Then the centre of mass of the body coincides with the centre of mass of the system and does not move. The axis of intermediate central moment of inertia of the body (axis $O^{\prime} \eta$ ) is also the axis of a principal central moment of inertia of the system. We assume that the principal central moments of inertia of the system are different from each other, and that $O^{\prime} \eta$-axis corresponds to the intermediate moment of inertia of the system (this is correct if masses of the moving particles are not too big). Seen in the body frame, the motion of the frame of the central principal axes of inertia of the system $O x y z$ is a rotation about the fixed $y$-axis.

Let $m$ be mass of each of the moving particles, $r$ be radius of the circle which is the trajectory of these particles, $\alpha=\alpha(\tau)$ be the polar angle of one of these particles in the plane of maximal and the minimal moments of inertia of the body. This angle is measured anti-clockwise from the axis of the minimal moment of inertia, $\alpha_{0}=\alpha(0)$. For the principal moments of inertia of the system we have $B=$ const and (see Appendix B)

$$
A(\tau)=\frac{1}{2}(A(0)+C(0))+\frac{1}{2} \sqrt{\Delta(\tau)}, \quad C(\tau)=\frac{1}{2}(A(0)+C(0))-\frac{1}{2} \sqrt{\Delta(\tau)},
$$

where $\Delta$ is expressed via components of the inertia tensor of the system in the body frame $I_{\xi \xi}=I_{\xi \xi}(\tau), I_{\zeta \zeta}=I_{\zeta \zeta}(\tau), I_{\xi \zeta}=I_{\xi \zeta}(\tau)$ as follows:

$$
\Delta(\tau)=\left(I_{\zeta \zeta}-I_{\xi \xi}\right)^{2}+4 I_{\xi \zeta}^{2}
$$


For components of the inertia tensor, the following formulas are derived in Appendix B:

$$
\begin{gathered}
I_{\zeta \zeta}=C(0)+2 m r^{2}\left(\sin ^{2} \alpha-\sin ^{2} \alpha_{0}\right), \quad I_{\xi \xi}=A(0)+2 m r^{2}\left(\cos ^{2} \alpha-\cos ^{2} \alpha_{0}\right), \\
I_{\xi \zeta}=2 m r^{2}\left(\cos \alpha_{0} \sin \alpha_{0}-\cos \alpha \sin \alpha\right) .
\end{gathered}
$$

Motion of particles causes a rotation of principal axes of inertia of the system with respect to principal axes of inertia of the body. This is the rotation around $O y$-axis with the angular velocity (see Appendix B)

$$
\omega_{*}=\frac{I_{\xi \zeta}\left(A-I_{\zeta \zeta}\right)\left(\dot{I}_{\zeta \zeta}-\dot{I}_{\xi \xi}\right)-\dot{I}_{\xi \zeta}\left(I_{\xi \zeta}^{2}-\left(I_{\zeta \zeta}-A\right)^{2}\right)}{\left(I_{\xi \zeta}^{2}+\left(A-I_{\zeta \zeta}\right)^{2}\right) \sqrt{\left(I_{\zeta \zeta}(t)-I_{\xi \xi}(t)\right)^{2}+4 I_{\xi \zeta}^{2}}} .
$$

Thus, seen from the frame $O x y z$, the body rotates about $O y$-axis with the angular velocity $-\omega_{*}$, and two particles move in a circle with the angular velocity $-\omega_{*}+\dot{\alpha}$. The $y$-component of the angular momentum of this motion is

$$
\varepsilon g_{2}=-B \omega_{*}+2 m r^{2} \dot{\alpha} .
$$

Then, according to formulas (2.5), (5.4),

$$
\rho=f_{2} G=B^{-1} g_{2} G=\varepsilon^{-1}\left(-\omega_{*}+\frac{2}{B} m r^{2} \dot{\alpha}\right) G .
$$

For a numerical illustration we take $\alpha=\alpha(\tau)=\alpha_{0}+\varepsilon t, A(0)=10.0, B(0)=8.0, C(0)=6.0$, $m=0.15, M=10.0, \alpha_{0}=0.2, r=2.5$, and $\varepsilon=0.0005$. The initial values of $G_{1}, G_{2}, G_{3}$ are 9.9, 9.0, 8.1, respectively.

Fig. 13 and Fig. 14 show the graphs of moments of inertia $A$ and $C$ vs $\tau$. (Note that $B$ is a constant.)

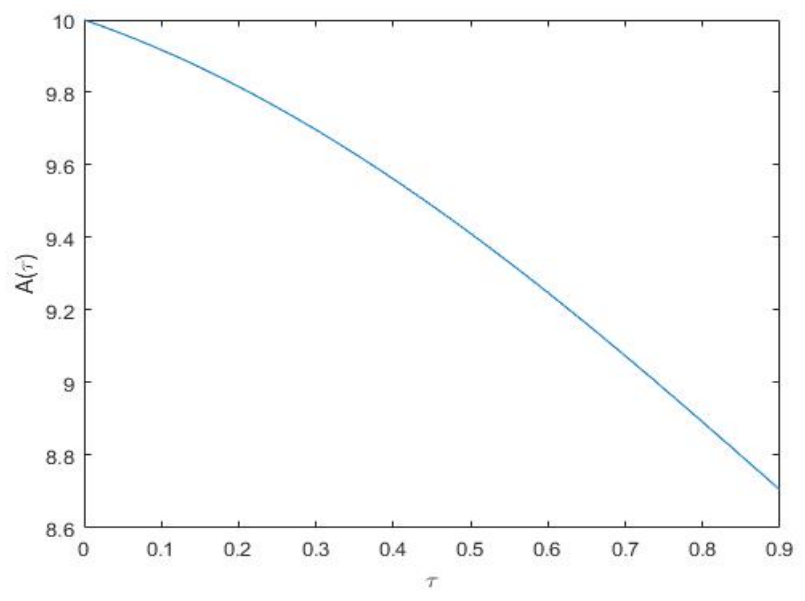

FIG. 13. Graph of the maximal moment of inertia $A$ vs $\tau$. 


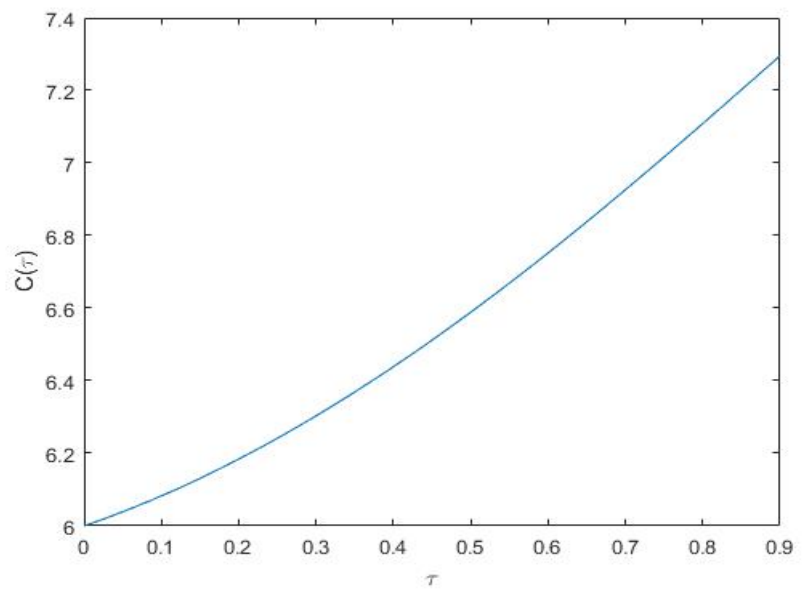

FIG. 14. Graph of the minimal moment of inertia $C$ vs $\tau$.

Fig. 15 and Fig. 16 show changes of $G_{1}$ and $G_{3}$ in dependence on the slow time $\tau$. These graphs demonstrate that a) the motion starts in domain $D_{1}$ and b) when $\tau$ is about 0.68 , the phase point crosses the separatrix several times and stays in domain $D_{2}$. Indeed, for the left part of these graphs $G_{1}$ takes both positive and negative values, and $G_{3}$ takes positive values. This corresponds to motion in $D_{1}$. For the right part of these graphs $G_{1}$ takes positive values, and $G_{3}$ takes both positive and negative values. This corresponds to motion in $D_{2}$. In the middle part of these graphs we see several passes through different domains.

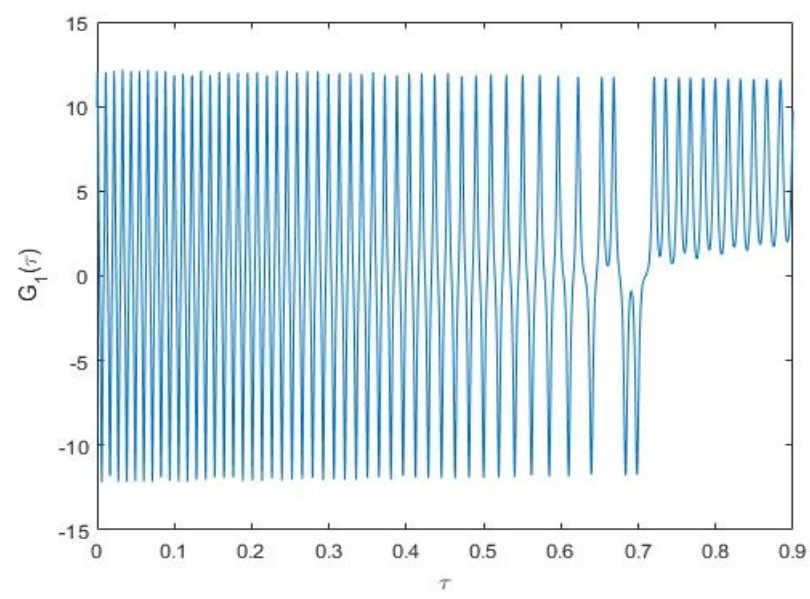

FIG. 15. Graph of $G_{1} v s \tau$. 


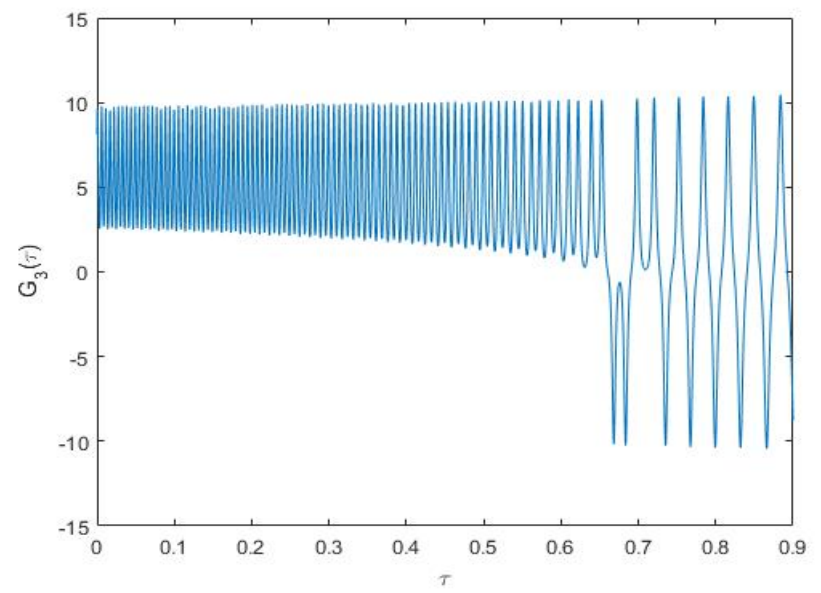

FIG. 16. Graph of $G_{3} v s \tau$.

Dependence of energy $H_{0}$ on slow time $\tau$ is shown in Fig. 17 (blue curve). The horizontal brown straight line corresponds to the value of energy on the separatrices. We see that the energy decays. It is equal to the energy along the separatrices when $\tau$ is around 0.68 , where the crossing happens.

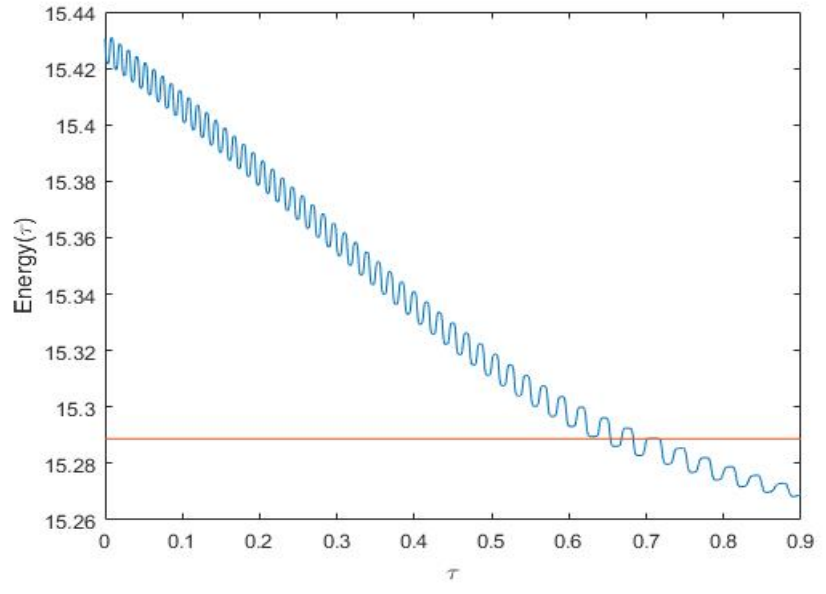

FIG. 17. Graph of energy $H_{0} v s \tau$.

Behaviour of action $I$ in slow time is shown in Fig. 18. We see that the action performs only small oscillations about a constant value. The amplitude of oscillations grows near the value of $\tau$ about 0.68 where the separatrix crossing occurs. 


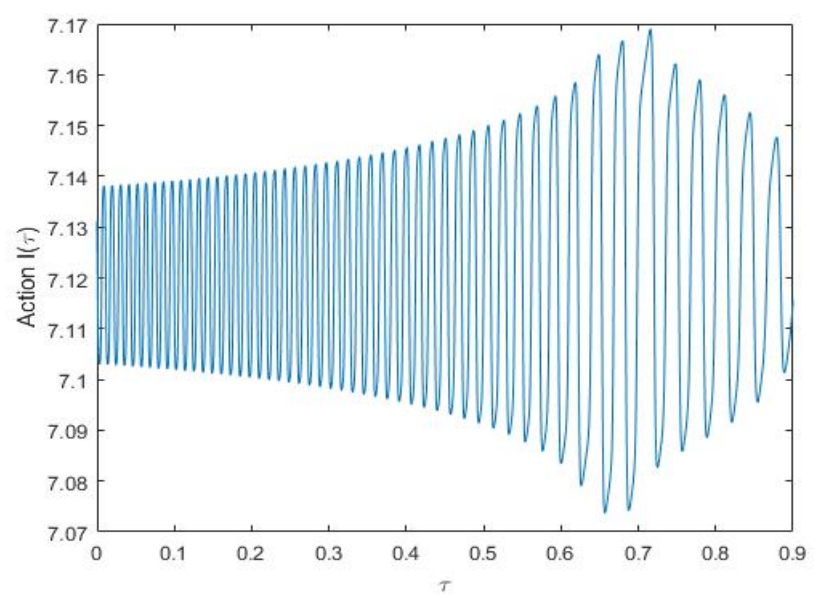

FIG. 18. Graph of action I vs $\tau$.

Fig. 19 shows the graph of area of the domain $D_{2}\left(\right.$ or $\left.D_{3}\right)$ vs $\tau$ (blue curve) and the initial value of action $I$ multiplied by $2 \pi$ (brown horizontal straight line). The blue and brown lines intersect at $\tau=0.683315$. This is the moment of separatrix crossing calculated in adiabatic approximation. This value is in a good agreement with moment of separatrix crossing seen in the previous pictures.

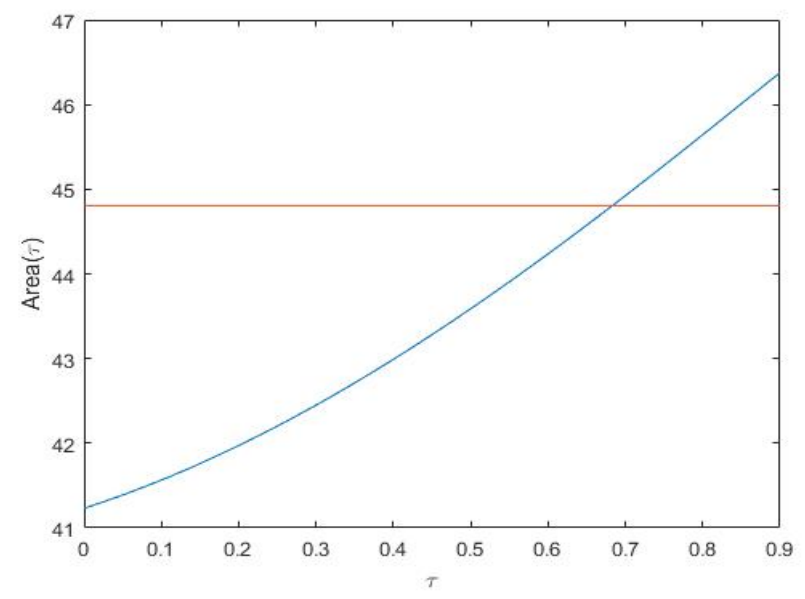

Fig. 19. Graph of area vs $\tau$.

To check the formula for probability we fix $G_{2}=9$ and consider 2000 initial conditions uniformly distributed in the square $9.85 \leq G_{1} \leq 9.95,8.05 \leq G_{3} \leq 8.15$. Trajectories with these initial conditions were captured into $D_{2}$ in 1574 cases and into $D_{3}$ in 426 cases. Thus, numerically calculated probability of capture into $D_{2}$ is $1574 / 2000=0.787$. For the moment of separatrix crossing $\tau_{*}=0.683315$ formulas (5.2), (6.2) give $\Theta_{*}=3.5006$ and $\rho_{*}=7.979405$. We have $3 \Theta_{*}<2 \rho_{*}<5 \Theta_{*}$. Thus we should use formulas (5.9) with $k=2$. We get $P_{1,2}=(-3 \cdot 3.5006+2 \cdot 7.979405) /(2 \cdot 3.5006)=0.77943$. We see that the numerical value for probability 0.787 is quite close to the result given by the theory. 
7 Conclusions We have described evolution of rotational dynamics of a body with a slowly varying geometry of masses using an adiabatic approximation. The separatrix crossing in the course of this evolution is associated with a probabilistic scattering of phase trajectories. We have calculated probabilities of different outcomes of the evolutions due to this scattering. These results could be useful in study of rotation of celestial bodies.

Appendix A. The model described in Sect. 6.1. Denote $\eta_{c}=\eta_{c}(\tau)$ the $\eta$-coordinate of the centre of mass $O$ of the system. Then, because the centre of mass of the body is $O^{\prime}$, we have

$$
\eta_{c}=\frac{m}{M} \eta .
$$

Denote $I_{\xi, \xi}$ the moment of inertia of the body about $\xi$-axis. For the maximal moment of inertia of the system $A(\tau)$ we obtain, using Steiner's theorem,

$A(\tau)=I_{\xi, \xi}+(M-m) \eta_{c}^{2}(\tau)+m\left(\eta(\tau)-\eta_{c}(\tau)\right)^{2}, \quad A(0)=I_{\xi, \xi}+(M-m) \eta_{c}^{2}(0)+m\left(\eta_{0}-\eta_{c}(0)\right)^{2}$.

Thus

$$
\begin{aligned}
A(\tau)-A(0) & =(M-m)\left(\eta_{c}^{2}(\tau)-\eta_{c}^{2}(0)\right)+m\left(\left(\eta(\tau)-\eta_{c}(\tau)\right)^{2}-\left(\eta_{0}-\eta_{c}(0)\right)^{2}\right) \\
& =\frac{(M-m) m^{2}}{M^{2}}\left(\eta^{2}-\eta_{0}^{2}\right)+m\left(\left(\eta-\frac{m}{M} \eta\right)^{2}-\left(\eta_{0}-\frac{m}{M} \eta_{0}\right)^{2}\right) \\
& =\frac{(M-m) m^{2}}{M^{2}}\left(\eta^{2}-\eta_{0}^{2}\right)+m\left(1-\frac{m}{M}\right)^{2}\left(\eta^{2}-\eta_{0}^{2}\right)=\frac{m(M-m)}{M}\left(\eta^{2}-\eta_{0}^{2}\right) .
\end{aligned}
$$

This implies the formula for $A(\tau)$ in Sec.6.1. Proof of the formula for $C(\tau)$ is completely analogous.

Appendix B. The model described in Sect. 6.2. The coordinates of the moving particles in the body frame $O \xi \eta \zeta$ are

$$
\xi= \pm r \sin \alpha, \eta=0, \zeta= \pm r \cos \alpha .
$$

Then the moments of inertia of the system can be written as

$$
\begin{gathered}
I_{\zeta \zeta}=I_{\zeta \zeta}(\tau)=C(0)+2 m r^{2}\left(\sin ^{2} \alpha-\sin ^{2} \alpha_{0}\right), \\
I_{\xi \xi}=I_{\xi \xi}(\tau)=A(0)+2 m r^{2}\left(\cos ^{2} \alpha-\cos ^{2} \alpha_{0}\right), \\
I_{\xi \zeta}=2 m r^{2}\left(\cos \alpha_{0} \sin \alpha_{0}-\cos \alpha \sin \alpha\right), \\
I_{\eta \eta}=B .
\end{gathered}
$$

Principal moments of inertia of the system are eigenvalues of the moments of inertia matrix:

$$
\hat{J}=\hat{J}(\tau)=\left(\begin{array}{ccc}
I_{\xi \xi} & 0 & I_{\xi \zeta} \\
0 & I_{\eta \eta}(t) & 0 \\
I_{\xi \zeta} & 0 & I_{\zeta \zeta}
\end{array}\right)
$$

The characteristic equation is

$$
\left(I_{\eta \eta}-\lambda\right)\left(\lambda^{2}-\left(I_{\zeta \zeta}(t)+I_{\xi \xi}(t)\right) \lambda+I_{\xi \xi}(t) I_{\zeta \zeta}(t)-I_{\xi \zeta}^{2}(t)\right)=0 .
$$


Then $I_{\eta \eta}=B$ is one of the principal moments of inertia. Other eigenvalues are

$$
\lambda_{1,2}=\frac{1}{2}\left(I_{\xi \xi}(t)+I_{\zeta \zeta}(t)\right) \pm \frac{1}{2} \sqrt{\left(I_{\zeta \zeta}(t)-I_{\xi \xi}(t)\right)^{2}+4 I_{\xi \zeta}^{2}(t)} .
$$

Then we have $I_{\zeta \zeta}+I_{\xi \xi}=A(0)+C(0)$.

Denote $\Delta=\left(I_{\zeta \zeta}(t)-I_{\xi \xi}(t)\right)^{2}+4 I_{\xi \zeta}^{2}$. Then

$$
A(\tau)=\lambda_{1}=\frac{1}{2}(A(0)+C(0))+\frac{1}{2} \sqrt{\Delta}, \quad C(t)=\lambda_{2}=\frac{1}{2}(A(0)+C(0))-\frac{1}{2} \sqrt{\Delta},
$$

which are formulas in Sect. 6.2.

Motion of the system frame with respect to the body frame is a rotation about $\eta$ axis with some angular velocity $\omega_{*}$. Formulas of the standard perturbation theory for eigenvectors of symmetric matrices (see, e.g., [3], Section 4.16) imply that

$$
\omega_{*}=\frac{\left(\dot{\hat{\jmath}}_{C}, \hat{e}_{A}\right)}{C-A} .
$$

Here $\hat{e}_{A}$ and $\hat{e}_{C}$ are unit vectors of axes $O x$ and $O z$ (eigenvectors of matrix (B.1)) and (.,.) denotes the standard scalar product. Explicit formulas for $\hat{e}_{A}$ and $\hat{e}_{C}$ are

$$
\hat{e}_{C}=\left(\begin{array}{c}
-\frac{I_{\xi \zeta}}{\sqrt{I_{\xi \zeta}^{2}+\left(\lambda_{1}-I_{\zeta \zeta}\right)^{2}}} \\
0 \\
\frac{\lambda_{1}-I_{\zeta \zeta}}{\sqrt{I_{\xi \zeta}^{2}+\left(\lambda_{1}-I_{\zeta \zeta}\right)^{2}}}
\end{array}\right), \quad \hat{e}_{A}=\left(\begin{array}{c}
\frac{\lambda_{1}-I_{\zeta \zeta}}{\sqrt{I_{\xi \zeta}^{2}+\left(\lambda_{1}-I_{\zeta \zeta}\right)^{2}}} 0 \\
0 \\
\frac{I_{\xi \zeta}}{\sqrt{I_{\xi \zeta}^{2}+\left(\lambda_{1}-I_{\zeta \zeta}\right)^{2}}}
\end{array}\right) .
$$

The explicit formula for $\omega_{*}$ is

$$
\begin{aligned}
& \omega_{*}=-\frac{1}{\sqrt{\left(I_{\zeta \zeta}(t)-I_{\xi \zeta}(t)\right)^{2}+4 I_{\xi \zeta}^{2}}}\left(\begin{array}{lll}
-\frac{I_{\xi \zeta}}{\sqrt{I_{\xi \zeta}^{2}+\left(\lambda_{1}-I_{\zeta \zeta}\right)^{2}}} & 0 & \left.\frac{\lambda_{1}-I_{\zeta \zeta}}{\sqrt{I_{\xi \zeta}^{2}+\left(\lambda_{1}-I_{\zeta \zeta}\right)^{2}}}\right)
\end{array}\right)\left(\begin{array}{ccc}
\dot{I}_{\xi \zeta} & 0 & \dot{I}_{\xi \zeta} \\
0 & \dot{I}_{\eta \eta} & 0 \\
\dot{I}_{\xi \zeta} & 0 & \dot{I}_{\zeta \zeta}
\end{array}\right)\left(\begin{array}{c}
\frac{\lambda_{1}-I_{\zeta \zeta}}{\sqrt{I_{\xi \zeta}^{2}+\left(\lambda_{1}-I_{\zeta \zeta}\right)^{2}}} \\
0 \\
\frac{I_{\xi \zeta}}{\sqrt{I_{\xi \zeta}^{2}+\left(\lambda_{1}-I_{\zeta \zeta}\right)^{2}}}
\end{array}\right) \\
& =\frac{I_{\xi \zeta}\left(A-I_{\zeta \zeta}\right)\left(\dot{I}_{\zeta \zeta}-\dot{I}_{\xi \zeta}\right)-\dot{I}_{\xi \zeta}\left(I_{\xi \zeta}^{2}-\left(A-I_{\zeta \zeta}\right)^{2}\right)}{\left(I_{\xi \zeta}^{2}+\left(A-I_{\zeta \zeta}\right)^{2}\right) \sqrt{\left(I_{\zeta \zeta}(t)-I_{\xi \xi}(t)\right)^{2}+4 I_{\xi \zeta}^{2}}}
\end{aligned}
$$

as it is given in (6.1).

Acknowledgments. The authors are thankful to A.V. Bolsinov and V.V. Sidorenko for useful discussions.

REFERENCES

[1] V. I. ARnol'D, Small denominators and problems of stability of motion in classical and celestial mechanics, Russian Mathematical Surveys, 18 (1963), pp. 85-191.

[2] V. I. Arnold, V. V. Kozlov, and A. I. Neishtadt, Mathematical aspects of classical and celestial mechanics, Springer-Verlag, 3 ed., 2006.

[3] R. Bellman, Introduction to Matrix Analysis, McGraw-Hill Book Co., 1960.

[4] A. V. Borisov and I. S. Mamaev, Adiabatic invariants, diffusion and acceleration in rigid body dynamics, Regular and Chaotic Dynamics, 21 (2016), pp. 232-248.

[5] F. L. Chernousko, L. D. Akulenko, and D. D. Leshchenko, Evolution of motions of a rigid body about its center of mass, Springer-Verlag, Chum, 2017. 
[6] A. DepRIT, Free rotation of a rigid body studied in the phase plane, American Journal of Physics, 35 (1967), pp. $424-428$.

[7] P. Goldreich and S. Peale, Spin-orbit coupling in the solar system, Astronomical Journal, 71 (1966), pp. 425438.

[8] P. Goldreich And A. Toomre, Some remarks on polar wandering, Journal of Geophysical Research, 74 (1969), pp. $2555-2567$.

[9] M. LARA AND S. FerRer, Integration of the Euler-Poinsot problem in new variables, 2010, https://arxiv.org/ abs/1101.0229.

[10] I. M. Lifshits, A. A. Slutskin, AND V. M. NabutovskiI, The scattering of charged quasi-particles from singularities in p-space, Soviet Physics Doklady, 6 (1961), pp. 238-240.

[11] K. W. Lips AND V. J. Mod, Three-axis attitude dynamics during asymmetric deployment of flexible appendages, Acta Astronautica, 8 (1981), pp. 575-590.

[12] I. Matsuyama, F. Nimmo, and J. X. Mitrovica, Planetary reorientation, Annual Review of Earth and Planetary Sciences, 42 (2014), pp. 605-634.

[13] W. Munk and G. J. F. MacDonald, The Rotation of the Earth, Cambridge University Press, New York, 1960.

[14] A. NeISHTADT, Passage through a separatrix in a resonance problem with a slowly-varying parameter, Journal of Applied Mathematics and Mechanics, 39 (1975), pp. $594-605$.

[15] A. NeishtadT, Averaging method for systems with separatrix crossing, Nonlinearity, 30 (2017), pp. 28712917.

[16] E. Routh, Dynamics of systems of rigid bodies, Part II, Macmillan \& Co, 1905.

[17] Y. SAdov, The action-angle variables in the Euler-Poinsot problem, Journal of Applied Mathematics and Mechanics, 34 (1970), pp. 922 - 925.

[18] K. TsuchiYa, Dynamics of a spacecraft during extension of flexible appendage, Journal of Guidance, Control, and Dynamics, 6 (1983), pp. 100-103.

[19] A. E. Zakrzhevskit, J. Matarazzo, and V. S. Khoroshilov, Dynamics of a system of bodies with programvariable configuration, International Applied Mechanics, 40 (2004), pp. 345-350. 